\title{
COMPONENTES ESTRUCTURALES Y FUNCIONALES DE LAS TUTORÍAS: UN CAMINO POR CONSTRUIR ${ }^{1}$
}

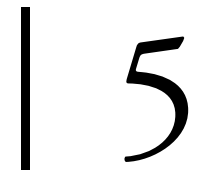

\section{Introducción}

La investigación que se presenta en este capítulo parte de las conclusiones a las que llegó un estudio anterior sobre la tutorías académicas, investigación que se adelantó en la Universidad Sergio Arboleda, y que, en particular, llama la atención en que éstas "no se prolongan y en la mayoría de las universidades las realizan como simples orientaciones en procesos discontinuos; en esta medida, reclama la urgencia de incluirlas en los planes de estudio universitarios" (Molina \& Gallardo, 2011, p. 42). A partir de ese trabajo surgieron los siguientes interrogantes: ¿Cuáles son las características estructurales y funcionales de un modelo de acompañamiento dirigido a estudiantes de instituciones de educación superior (IES)? y ¿̨cuál debería ser el modelo de acompańamiento apropiado para mejorar las condiciones educativas y de formación integral de los estudiantes universitarios?

Con el propósito de responder estas preguntas, en esta investigación se planteó como objetivo proponer un modelo de tutorías estructural y funcional orientado a los estudiantes de las IES, para lo cual es primordial identificar los motivos institucionales y académicos que tienen estas instituciones para desarrollar los programas de acompañamiento tutorial. Asimismo, es necesario analizar la incidencia que tienen los estudiantes beneficiados con acompańamientos en la vida universitaria y, en esa medida, establecer la relación entre las características funcionales y estructurales de los modelos de tutorías y su correspondencia.

Respecto a los supuestos que fundamentan el estudio, cabe destacar que se consideró que los programas de tutorías estructurales y funcionales en las IES están encaminados a prevenir la deserción y que los programas de acompańamiento permiten a los estudiantes obtener mejores resultados académicos, apropiarse de su formación y encaminarse en su quehacer profesional.

Ahora bien, el proyecto aplicó una metodología cualitativa, la cual, según Hernández, Fernández y Baptista (2006), no busca la réplica, sino que se conduce

1 Este capítulo corresponde a la finalización de la segunda fase del proyecto marco de investigación que desarrolló el Grupo de Investigación Educativa de la Universidad Sergio Arboleda (Invedusa) sobre Tutorías Académicas. 
en un ambiente natural, su proceso es recurrente, inductivo y analiza las realidades. Entre sus bondades están la profundidad de ideas, su amplitud, la contextualización que se puede hacer de los fenómenos representativos y a la vez la riqueza interpretativa.

En correspondencia con esta orientación metodológica, la investigación contó con un observador que se acercó a un sujeto real de cada una de las IES seleccionadas y que recolectó sistemáticamente información de sus experiencias y opiniones sobre el tema específico de los programas de acompańamiento a estudiantes universitarios. Asimismo, con base en las reflexiones del observador se describió y caracterizó socialmente la situación de los programas de tutorías. A continuación, se sintetizan las principales características de los estudios cualitativos que orientaron está investigación:

- Holístico: Desea comprender la situación de las instituciones de educación superior por medio de la descripción de las acciones tutoriales que estas desarrollan.

- Naturalista: El trabajo de campo busca comprender el funcionamiento de los programas de acompañamiento y su relación con la institucionalidad de la IES.

- Interpretativa: Analiza el punto de vista de los estudiantes y docentes participantes y cómo estos se relacionan con la filosofía y estructura institucional.

- Empática: Busca establecer las características de los programas de acompañamiento, las experiencias que se derivan de estos y sus actores: estudiantes, docentes e IES. (Rodríguez \& García, 1996)

Consecuentemente, el método empleado es fenomenológico, el cual se estructura en tres etapas: Descripción, estructuración y, por supuesto, discusión. En descripción se siguen los siguientes pasos: Elección de la técnica o procedimiento, la aplicación de la técnica seleccionada y el diseño de la descripción protocolar (Trejo, 2012). Específicamente, en esta fase el estudio tuvo como objetivo describir las experiencias de los programas de tutorías de las IES y comprender los procesos educativos y formativos que estos sugieren. Con este propósito se seleccionó la entrevista como herramienta de obtención de información, la cual permitió conocer el rol del tutor y la funcionalidad e impacto que estas acciones tienen para la vida universitaria.

Los cuestionarios que se usaron partieron de la referencia teórica y experiencial sobre el concepto y la funcionalidad de las tutorías para el desempeño académico 
y la formación integral de los estudiantes de pregrado. Al respecto, es importante resaltar que los análisis de los datos que arrojó este estudio fueron compartidos con las IES participantes y los actores de los programas de acompañamiento, lo cual les ha permitido realizar una evaluación de la funcionalidad e institucionalidad de las acciones tutoriales, tal como lo expresa Rodríguez y Vallderiola (2007).

Durante la entrevista se utilizaron cinco tipos de preguntas: (1) Las demográficas o biográficas, que indagan su trascendencia en el programa de tutorías; (2) las sensoriales, relativas a lo que percibe con los estudiantes durante y luego del acompańamiento; (3) las experienciales, que exploran la experiencia docente como tutor y el perfil que, en su criterio, se debe tener para desarrollar esta función; (4) las preguntas sobre sentimientos buscan reconocer la existencia o no de un vínculo afectivo con el estudiante gracias a la atención de las tutorías y, finalmente (5) las de opinión, que tienen el fin de conocer el modo en que valoran determinadas situaciones (Rodríguez \& Vallderiola, 2007).

Los datos se codificaron teóricamente para conceptualizarlos y construir teorías fundamentadas que permitan describir las variables del modelo de tutorías en relación con la dimensión estructural (institucional: misión, currículo, plan de estudios y perfil del docente) y a la funcional (programas de acompañamiento: concepto, funciones, metodología y papel del tutor). Además, primero se analizó individualmente cada institución y posteriormente se correlacionó la información de las tres IES para determinar similitudes o contrastes con el objetivo de fundamentar un modelo que brinde una mirada integral a los jóvenes inmersos en la educación superior.

En este último paso se trianguló la información, pues de esta forma es posible que el investigador adopte diferentes perspectivas sobre el problema central de estudio y, de esta manera, responda las preguntas de investigación y produzca conocimiento en diferentes niveles. Como sugiere Flick, (2014), la triangulación se organizó gráficamente por medio de un mapa mental.

\section{Los programas de acompañamiento}

Las tutorías han sido definidas como acciones pedagógicas que tratan de favorecer las condiciones académicas y aportar en el proceso de enseñanza y aprendizaje de manera individual y grupal. Sin embargo, según Monge (2009), la función tutorial no solo debe ser un elemento inherente a la función educativa. Estas, a su vez, son parte esencial del desarrollo curricular, pues permiten la orientación para la 
formación profesional y personal de los estudiantes. Se trata de un proceso dirigido a comprenderlos desde las diferentes ópticas y dimensiones y en el cual se desarrollan actividades de orientación y asesoría.

Por su parte, los docentes que participan en estos programas de acompańamiento amplían su accionar gracias a que salen del aula y de la cotidianidad de los cursos establecidos, intencionan sus expectativas y las encadenan con los objetivos educativos. Como sostiene Monge (2009), "ser un buen docente es ser un buen profesor y un buen tutor uniendo estrechamente los tiempos y objetivos que perseguimos en la clase y el despacho" (p. 14). De esta manera se crea y fomenta una cultura en la que el estudiante participa activamente en su proceso educativo con base en una relación dialógica con los profesores.

De acuerdo con este esbozo, durante los últimos años en las IES de Colombia han crecido los programas de acompañamiento a estudiantes, como parte de las estrategias enfocadas a permitir la mejor adaptación a la vida universitaria, a la calidad educativa y a aumentar, en esa medida, los porcentajes de permanencia de los estudiantes. Muchos de estos proyectos estructuran acciones y generan espacios de atención académica para que los estudiantes aclaren dudas sobre algunas temáticas, mientras que otras instituciones le han apostado a complementar estas atenciones con actividades enfocadas a hacer un acompañamiento de índole personal, como el coaching, el trabajo entre pares, la mentoría, la asesoría personalizada, entre otros.

Por esta vía se han establecido vínculos de confianza entre tutores y tutorados, en los cuales el profesor tiene la oportunidad de intervenir en los procesos con un enfoque humanista e integral. En consecuencia, el docente evalúa cómo aprenden sus estudiantes, reflexiona de qué manera podría potenciar significativamente sus aprendizajes y, así, puede comprender los escenarios que le configuran desde todas las dimensiones del ser. Por su parte, cuando el tutorado recibe el apoyo de un adulto significativo en su vida universitaria, desarrolla y fortalece capacidades como la crítica, reflexiva y propositiva, así como la toma de decisiones, lo cual no solo aumenta su autoestima y autodeterminación, sino que además, a la vez, genera mayor apropiación de su formación y permanencia en el ámbito universitario.

Por esta razón es vital estudiar los programas de acompańamiento, que generalmente han recibido el nombre de tutorias. Estas tienen su origen en las Universidades de Oxford y Cambridge en el siglo XIX, donde surgió la necesidad de que los estudiantes aprendan a aprender (Sánchez, 2006). Las tutorías pueden definirse como espacios educativos de aprendizaje interpersonal, basados en el trabajo cooperativo y la interacción entre sujetos interesados en el saber, un escenario que 
crea la oportunidad de establecer relaciones de confianza para reforzar las capacidades y las habilidades que los motivarán a continuar sus estudios. No obstante, es necesario revisar esta conceptualización a la luz de la revisión bibliográfica, ya que la tutoría se puede concretar de acuerdo con sus categorías, modalidades del rol docente y funcionalidad, tal y como se describe a continuación:

- Tutoría por categorías. Se dividen en cuatro escenarios, en los cuales la tutoría se define como: (1) Espacio de actividad académica donde se detectan las dificultades y los logros; (2) espacio de interacción donde existe el intercambio de saberes; (3) acción didáctica, vista como la relación pedagógica. (4) Estrategia metodológica, pues posibilita encuentros institucionales periódicos con un tutor que actúa como guía (Castillo, Torres \& Polanco, 2009, p. 7).

- Modalidades. Se entiende desde según (1) el modo de realizarse: Presencial y a distancia. (2) El material: Telefónica, correspondencia, telemática, radial y audiovisual. (3) El propósito: Inducción, eventos formativos, información de retorno, académico administrativo y (4) el número de estudiantes: se puede adelantar de manera personalizada o en grupo (Ladino, 2004, p. 89). La tutoría presencial permite construir hábitos que desarrollan la autonomía en procesos de aprendizaje, mientras que la tutoría grupal tiene la ventaja de adelantar el conocimiento a través de la multiculturalidad, el aprendizaje interpersonal y por medio del trabajo cooperativo y de apoyo mutuo.

- Rol del docente. Debido a que la tutoría es una atención extra-aula, se convierte en una estrategia educativa en la que el docente vigila los procesos de enseńanza en el aula y las particularidades de aprendizaje del estudiante. El docente, por lo tanto, no solo se dedicará a transmitir conocimiento en el aula, sino que la tutoría se convertirá en una modalidad de su actividad que involucra un conjunto sistematizado de acciones educativas centradas en el estudiante y que permite diversos niveles y formas de intervención. Al respecto, Peinado et al. (2011) retoman las palabras de Bagnis Garibay (2003):

Por otro lado, la tutoría es el proceso de ayuda u orientación al alumno o al grupo que el profesor tutor debe realizar, además y en paralelo a su propia acción como docente. Esta es, pues, la orientación; pero desde la perspectiva y posibilidades de ser realizada por los propios profesores, equivale a una orientación a lo largo de todo el proceso educativo, para 
que el alumno supere su rendimiento académico, solucione sus dificultades escolares y logre hábitos de trabajo y estudio. (p. 24)

- La tarea del docente, por lo tanto, es promover capacidades como la toma de decisiones y la resolución de problemas. Por su parte, el estudiante siente el apoyo de su profesor y se motiva a capacitarse, a explorar sus aptitudes, a mejorar su aprendizaje y proyectar de manera más responsable su futuro.

- Funcionalidad. A través de la tutoría se resuelven dudas y refuerzan conocimientos, pero, a la vez, se avanza en el desarrollo de una metodología de estudio y se mejora la actitud hacia el aprendizaje, de tal forma que se estructura un acompańamiento para mejorar los desempeńos académicos. Pero esto no queda allí, la tutoría fortalece la formación integral de los estudiantes, quienes refuerzan su autoestima y seguridad personal cuando desarrollan sus habilidades intelectuales, pues asumen sus responsabilidades de manera más autónoma.

Aunque la tutoría se ofrece a toda la comunidad estudiantil, se debería utilizar principalmente para proporcionar enseñanza complementaria a los estudiantes con dificultades, con potencialidades, o con necesidades especiales. Así, la tutoría no solo es una herramienta para la preparación académica y formación integral de los estudiantes, sino que también se puede convertir en una oportunidad para actuar frente a la prevención de problemas de adaptación al medio universitario, pérdida académica y/o deserción, así como una medida de intervención para atender problemas de aprendizaje, lectoescritura, ansiedad ante exámenes, estabilidad emocional, proyección de carrera, entre otros, como lo afirman Peinado et al. (2011):

La tutoría se convierte, entonces, en una vía de creación de nuevos y múltiples conocimientos, que posibilita visualizar al estudiante desde una perspectiva integral y diferenciada, más allá de patrones uniformes y, por tanto, puede conducir a resultados educativos más satisfactorios tanto para los estudiantes como para los docentes. (p. 24)

Desde esta perspectiva, los autores presentan el proceso del programa de acompañamiento, como se evidencia en la figura 1. 

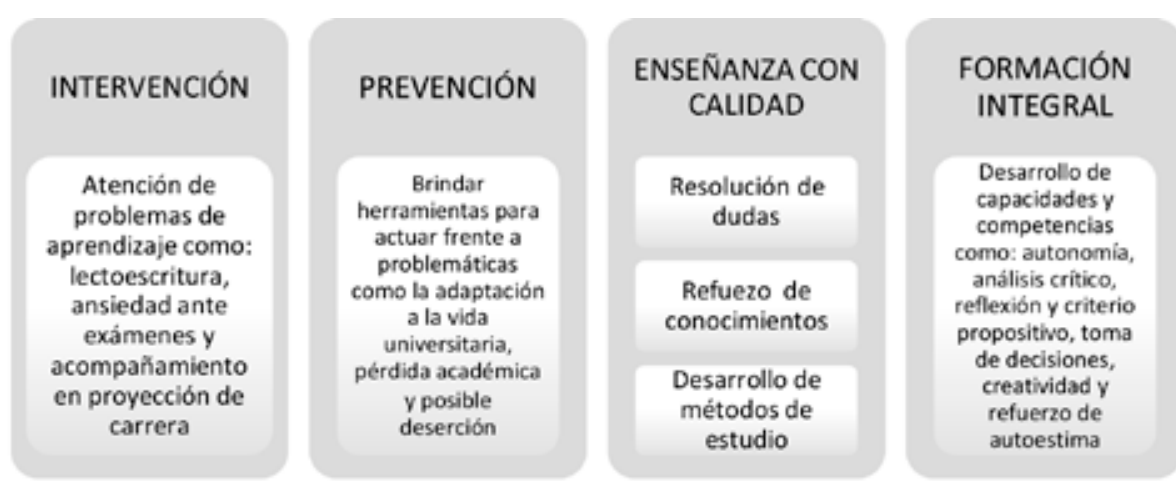

\section{Programa de acompañamiento}

Figura 1. Funcionalidad de la tutoría

Fuente: Peinado, Huerta, Mendoza y Ladrón (2011, p.25).

Los conceptos que expone la figura 1 pueden dar cuenta de por qué los programas de acompañamiento han sido modelados en cada institución de acuerdo con sus expectativas e intereses para resolver algunas de las necesidades de la población estudiantil.

\section{Experiencias de otras Universidades.}

Ahora bien, entre las experiencias que se pueden recalcar en Latinoamérica se encuentra la de la Universidad de Quilmes, en Argentina. Esta institución implementó un sistema basado en cuatrisemestres, durante los cuales el estudiante recibe un acompañamiento permanente desde los primeros semestres hasta la finalización de su plan de estudios. Este seguimiento a su proceso les permite adaptarse a la vida universitaria, tener apoyo pedagógico y recibir asesoría en cuanto a sus prácticas empresariales y proyección de estudios de posgrado (Molina, 2012).

En Argentina también es conocido el caso de la Universidad Tecnológica Nacional de Haedo, la cual implementó un sistema de tutorías para mejorar los índices de retención y de graduación que se basan en un acompañamiento integral que motive a los estudiantes a reconocer sus necesidades académicas y aspiraciones profesionales, gracias a lo cual logran un mejor desempeño académico (Rodríguez \& Sierra, 2014). 
Otro referente latinoamericano que ha cumplido la inclusión institucional de los programas de tutorías es México, específicamente, la Universidad Autónoma de Hidalgo, tal como se sintetiza en el V Encuentro Nacional de Tutorías Valles, Torres \& Godínez, 2012. En esta IES, la tutoría opera como sustento en aspectos académicos, económicos, sociales y personales, pues ofrece asistencia al estudiante a lo largo de su trayectoria escolar a través de un modelo de tutoría integral, con apoyo de un asesor psicológico, un trabajador social y un maestro orientador.

La Universidad de Guadalajara, por su parte, realiza un estudio minucioso que determina las asignaturas con mayor reprobación y las posibles falencias que la ocasionan para promover la ayuda entre pares e implementar estrategias que permitan mejorar el desempeño académico por medio de equipos de trabajo liderados por estudiantes.

Finalmente, vale la pena recalcar la experiencia de la Universidad Autónoma del estado de México, que creó un programa que incorporó inicialmente doce tutores, a quienes se les asignaron quince estudiantes pertenecientes a las licenciaturas de comunicación, sociología y ciencias políticas. Este proceso tiene como objetivo desarrollar acciones tutoriales para así atender al estudiante en diferentes contextos y apoyarle desde sus propias características.

A medida que el programa fue creciendo, en la Universidad Autónoma se incorporó un sistema automatizado llamado Sitauaemex, que consiste en un espacio de comunicación para apoyar la atención oportuna, orientación y asesoría, así como para incrementar el aprovechamiento de los espacios tutoriales y garantizar el rescate académico. Este proceso tiene tres momentos fundamentales: (1) Capacitación de tutores, (2) revisión del rendimiento académico de cada tutorado para orientar su trayectoria académica y disminuir el rezago estudiantil, y (3) generar las alternativas necesarias para desarrollar el aprendizaje autónomo seleccionando el material de lectura, los ejercicios, los hábitos de estudio, las técnicas de lectura respectivos, entre otros.

Entre las conclusiones del este estudio de la Universidad Autónoma se pueden mencionar las siguientes:

a. La acción tutorial con énfasis en la formación integral contribuye al desarrollo del conocimiento, favorece el clima de clase, contribuye a la madurez vocacional y desarrolla habilidades y estrategias de aprendizaje, lo cual permite, a la vez, que la docencia influya en valores y actitudes éticas.

b. Para que un programa de tutorías funcione realmente, es necesario que los actores involucrados participen de forma activa y persigan el mismo objetivo (tutores, coordinador, tutorados, autoridades). 
c. La tutoría es el espacio para fomentar capacidades como el análisis crítico, la reflexión, la creatividad y el criterio propositivo.

Asimismo, se determinó que cerca del 67,6 \% de los espacios realizados durante el periodo de evaluación trataron temas de índole académico, en un segundo lugar complementado con aspectos personales. En el 2009, los estudiantes afirmaron que no deseaban asistir a las tutorías porque consideraron que la atención del tutor no daba respuesta a sus expectativas, así que solo acudían porque es un requisito para la inscripción del siguiente semestre. Esta situación invita a reflexionar sobre la importancia que tiene el desempeńo del tutor y la relación que establece con el tutorado, ya que, al parecer, sería el fundamento central para el cumplimiento del objetivo de los programas de acompańamiento (Gómez, 2012).

Pasando al ámbito educativo nacional, universidades colombianas como la Pontificia Universidad Javeriana, La Salle, la Pontificia Bolivariana, El Rosario, El Bosque, la Universidad Sergio Arboleda, entre otras, han implementado programas de tutorías ofertados como servicios educativos y los han incluido como parte del modelo pedagógico, con lo cual buscan fortalecer el perfil académico y la participación asertiva de los estudiantes en los diferentes escenarios universitarios.

Entre las universidades destacadas en el ámbito local se encuentra el programa de tutorías de la Universidad del Rosario, denominado "Apoyo al desarrollo integral del estudiante rosarista”, el cual actúa como un comité institucional contenido en el Plan Integral de Desarrollo. El objetivo central de este Programa Pacto es mejorar el rendimiento académico y promover la autonomía y la responsabilidad de los estudiantes para reducir los índices de deserción (Rodríguez \& Sierra, 2014).

En este contexto, algunos autores han investigado la manera en que las instituciones educativas deben desarrollar los planes de acciones tutoriales - Plan de Acción Tutorial (PAT) — . Este se refiere al diseño de un proceso que tiene como fin contribuir al desarrollo integral de los estudiantes y que, además, se inserta en la actividad educativa de manera sistemática e intencional mediante la implementación de estrategias de orientación y de acompañamiento (Castillo, Torres \& Polanco, 2009).

De acuerdo con Delgado (2005), para planificar y desarrollar adecuadamente el PAT se deben tener en cuenta cinco características, las cuales se presentan a continuación:

1. Diseño básico. En este se concretan los requerimientos mínimos para el diseño del PAT. En primer lugar, se tiene la conformación de un grupo 
de participantes: (1) Tutores con buena actitud y el compromiso de atender a los estudiantes, que estén dispuestos a promover las tutorías como una herramienta que responde a sus expectativas y necesidades. (2) Coordinador del programa, quien tiene claras sus funciones, entiende y promueve el objetivo del programa y, (3) el grupo de estudiantes universitarios inscritos en los programas de las facultades participantes.

En segundo lugar, es primordial obtener la información necesaria en una base de datos o listado de estudiantes por licenciatura con el fin de realizar la asignación de tutores y el calendario de reuniones tutorados-tutores que permitirá hacer el respectivo plan de trabajo.

2. Plan de trabajo. Para definir el plan de trabajo se recomienda tener en cuenta que las acciones tutoriales sean diseñadas de acuerdo con una evaluación previa de las necesidades y expectativas de los estudiantes, así como teniendo en cuenta todas sus dimensiones (personales, académicas y profesionales). Posteriormente, se deben establecer los aprendizajes concretos que se van a desarrollar, como son los que están centrados en la actividad académica y las estrategias para el mejoramiento de habilidades, el desarrollo de competencias y la adquisición de actitudes y valores. Igualmente, es importante considerar si el plan de trabajo propuesto contiene o no un valor crediticio, pues si no lo tiene, puede generar la sensación en los estudiantes de que no es una actividad curricular.

3. Momentos de intervención en la vida universitaria. El primer momento de intervención del PAT tiene lugar cuando los estudiantes ingresan a la universidad, etapa en la cual se brinda información y asesoría sobre los servicios que ofrece la institución, la composición de las facultades, sus funciones, las características del plan de estudios, la conformación de la trayectoria académica, el sistema de becas, entre otros. Además, se ofrecen cursos de orientación sobre sistemas, inglés, métodos de estudio, redacción y presentación de trabajos de investigación.

En el segundo momento para el desarrollo académico los tutores deberán acompañar a los estudiantes en los aspectos académicos, orientarlos sobre las prácticas profesionales, el trabajo de investigación final y apoyar la toma de decisiones en la proyección laboral.

Finalmente, el tercer momento corresponde a la finalización de los estudios. En esta etapa definitiva, los estudiantes reciben orientación para que definan sus estudios de posgrado, técnicas para la solicitud de trabajo (hoja de vida, entrevista) y otros aspectos relevantes a la formación profesional. 
4. Recursos. Para el funcionamiento adecuado de los PAT las instituciones deberán contar con recursos humanos, financieros, materiales y de servicios técnicos idóneos y suficientes.

5. Evaluación. La implementación del plan se lleva a cabo por medio de reuniones en las cuales se organiza su planeación, seguimiento y evaluación. Específicamente, la evaluación tiene como fin revisar si los objetivos con los cuales se implementó el plan se han cumplido y, en esa medida, definir el plan de mejoramiento continuo. Es conveniente realizar la evaluación al inicio, durante y al final del proceso, así como tener en cuenta que los indicadores deben estar orientados a responder las necesidades y las problemáticas concretas de los estudiantes que han sido detectadas previamente por medio de un estudio diagnóstico.

Además de estas cinco características, Castillo, Torres y Polanco (2009) sostienen que el PAT debe articular y plantear de forma coherente las acciones de los tutores y la propuesta curricular ofertada. Así mismo, permitir procesos de enseñanza-aprendizaje adaptados a las necesidades de los estudiantes y que favorezcan su integración en los grupos, de tal manera que se contribuya a mantener un clima de aprendizaje y desarrollo personal adecuado. Finalmente, recomiendan establecer una relación con los grupos familiares basada en la colaboración (sobre todo en las instituciones de educación básica y media).

Ahora bien, para lograr una mayor eficiencia del PAT es fundamental realizar una planeación funcional y operativa, con acciones realizables y realistas que cuenten con la participación reflexiva de los demás agentes de educación. Se necesita trabajo en equipo para implicar a los miembros del programa, quienes se deben basar en la humanización del sistema educativo y, a la vez, favorecer las relaciones entre la comunidad educativa (Castillo, Torres y Polanco, 2009).

Como se puede observar, en esta descripción se reflejan las diferentes modalidades y funcionalidades de los programas de tutorías, que en la mayoría de las ocasiones son acciones sin relación directa con las IES y que carecen de políticas organizacionales que permitan generar un modelo estructural y funcional, el cual es, precisamente, el objeto central de este estudio.

En consecuencia, es importante que las directivas académicas, los docentes y el personal de Bienestar Institucional de las diferentes instituciones seleccionadas conozcan las características de los programas de tutorías, el impacto académico en la población estudiantil y las transformaciones que estas permiten. Así, un buen modelo logrará proyectar acciones continuas en pro de evitar, por ejemplo, la deser- 
ción estudiantil, detectar las potencialidades y las fortalezas de sus estudiantes, en últimas, avanzar en los caminos de la alta calidad académica de las IES del país.

En ese sentido, proponer un modelo de acompańamiento con base en los hallazgos de las IES constituye un referente interinstitucional que motiva a otros centros de educación a implementar estrategias que apoyen la transición de la vida escolar a la universitaria y que promuevan su permanencia en programas de educación superior. Precisamente, analizar y describir las acciones de un modelo de Programa de Tutorías funcional y estructural constituye parte del aporte teórico de esta investigación, toda vez que "es un tema relevante dentro del ámbito educativo en el contexto universitario que ocupa un lugar destacado a nivel nacional e internacional, tanto en Europa, como en América Latina y el Caribe" (Rodríguez $\&$ Sierra, 2014, p. 30). Asimismo, es un tema innovador en Colombia que está causando curiosidad en los investigadores académicos y en el campo de la psicopedagogía, especialmente ante la aparición de beneficios gubernamentales como el programa de créditos condonables Ser Pilo Paga.

\section{Resultados}

Los resultados describen el estado actual de los programas de acompañamiento de las tres IES participantes: la Universidad de Antioquia, la Universidad del Rosario y la Universidad Sergio Arboleda. Así mismo, exponen la triangulación que se hizo desde la voz de los directores de programa y de un profesional que lo ejecuta. Cabe señalar que estas descripciones dan cuenta de las cuatro categorías básicas del modelo que se propone: (1) Características del programa, (2) intención de los programas de acompañamiento, (3) beneficios para el estudiante y (4) perfil del tutor.

\section{Estado actual de los programas de acompañamiento de las IES participantes}

\section{Universidad del Rosario: Pacto, una cultura de acompañamiento}

El programa de la Universidad del Rosario, denominado Pacto (Programa de Acompańamiento para Todos), actúa como una política institucional que fue creada en el año 2005. Organizacionalmente, depende de la Dirección de Estudiantes, la cual es parte de la Vicerrectoría de la IES y su objetivo es brindar asesoría y acompañamiento a la población estudiantil en todas las instancias de su vida universitaria. 
El programa cuenta con el liderazgo de un director de cohorte para cada facultad o escuela, quien cumple la función de atender a los estudiantes por medio de diferentes encuentros programados. Cada unidad de la universidad decide con qué intensidad y frecuencia utiliza los servicios que se ofrecen.

Teniendo en cuenta que los estudiantes necesitan especial atención durante los primeros semestres luego de que han ingresado a la educación superior, Pacto diseñó una metodología de atención para la población del primer semestre, como se muestra en la figura 2.
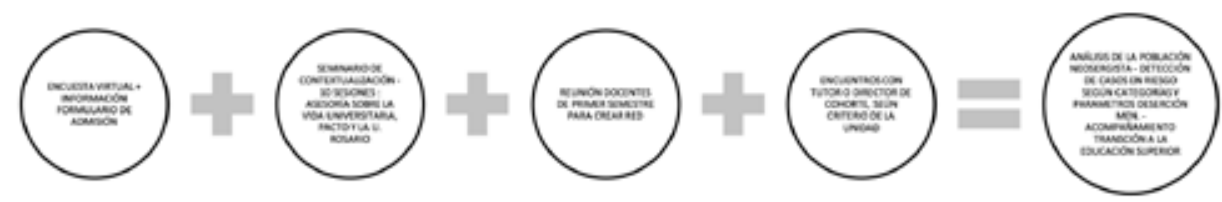

Figura 2. Acompañamiento y caracterización a estudiantes de primer semestre en la Universidad del Rosario

Fuente: Elaborada por los autores.

Como se observa en la figura 2, el programa aplica una encuesta virtual para elaborar un primer diagnóstico. Posteriormente, en la semana uno, realiza el Seminario de Contextualización, el cual comprende un espacio de diez sesiones donde los neorrosaristas reciben información y asesoría en relación con las herramientas necesarias y las prácticas para transitar adecuadamente del colegio a la vida universitaria (proyecto de vida, cómo citar según normas APA, conocimiento del reglamento, hábitos de estudio, conocimiento de Pacto, tramitología, etc.). Este escenario no solo constituye una oportunidad para conocer y acercarse a los estudiantes, sino que además hace posible que se generen alertas tempranas. Posteriormente y durante todo el semestre, se llevan a cabo reuniones preventivas y de asesoría individual. Con estos insumos — la información derivada de la fase de admisión, el cuestionario y el seminario de contextualización — la institución analiza la situación de cada estudiante y prioriza los casos en riesgo de pérdida o abandono, de acuerdo con los parámetros establecidos por los informes de deserción del Ministerio de Educación Nacional. De hecho, la universidad ha adelantado gestiones para comprar un software que permita detectar más rápidamente los casos para hacer seguimiento.

Otra característica sobresaliente de Pacto es su compromiso de generar una cultura de acompañamiento en cada miembro de la organización. En palabras de su director, Aldo Buenaventura, 
se pretende que cada uno de los colaboradores de la IES estén preparados para generar alertas tempranas, como son los conductores de los buses, el personal de servicios generales, el área administrativa... para que así el programa sea visto como el lugar donde se quiere acompañar al estudiante, no como un ente fiscalizador. Así, entonces, el personal de la IES está instruido para que en caso de que se presente una situación que involucre a un estudiante y que afecte su bienestar, se acerque al director de cohorte de cada facultad o escuela.

En este escenario es importante mencionar la experiencia de la Escuela de Medicina, la cual acoge programas académicos como medicina, fisioterapia, fonoaudiología, terapia ocupacional e ingeniería biomédica. En este caso, la Universidad del Rosario ha detectado que la mayoría de los estudiantes desertan por causas académicas, es decir, hábitos de estudio, métodos de trabajo, manejo de tiempo académico y la identificación de las formas de aprendizaje que supone el ingreso a la vida universitaria.

En respuesta a este diagnóstico, la institución definió mecanismos particulares para hacer el acompañamiento de esta población. Por ejemplo, aunque la Escuela tiene una directora de cohorte, cada programa cuenta con un tutor o coordinador de tutorías, quienes realizan una reunión mensual para acordar el plan de acompañamiento que se va a desarrollar, identificar casos puntuales de estudiantes que están en riesgo de pérdida o deserción y definir los posibles talleres que se van a desarrollar en beneficio del desempeño académico y/o desarrollo personal.

Por su parte, en cada programa el tutor orienta al estudiante en los siguientes aspectos: La inscripción de asignaturas de acuerdo con la administración de la malla curricular, trámites administrativos y las áreas a las que debe acudir; además, los refiere a los profesores del programa y les indica los horarios de monitorias y/o de atención docente. Específicamente, a los estudiantes del programa de medicina se les asignan un tutor que los acompaña desde el día de inducción hasta la graduación.

\section{Universidad Sergio Arboleda: Oferta de servicios para el bien- ESTAR ESTUDIANTIL}

En la Universidad Sergio Arboleda, los programas de acompañamiento están a cargo de la Decanatura de Estudiantes y existe un portafolio de servicios que permite que los estudiantes cuenten con apoyo en diferentes áreas y así dar una mirada integral a su formación.

De hecho, las estrategias de acompañamiento están dispuestas desde el momento de la admisión, especialmente con la población que tiene condiciones particulares, como los becados y en condición de discapacidad (tejido humano). 
Con este propósito, la institución realiza una entrevista presencial y elabora una caracterización que le permite establecer el tipo de acompañamiento que requieren $y$, posteriormente, hacer seguimiento con notas de primer corte. Una vez han ingresado a primer semestre, los estudiantes reciben información respecto a los servicios de la decanatura durante la inducción y luego en el taller Desafío U, espacio en el cual se realiza una encuesta adicional de caracterización.

El portafolio de acompañamiento cuenta con los siguientes servicios que están dirigidos a las personas matriculadas y egresadas, quienes deben acercarse a la decanatura para acceder a ellos:

- Acompañamiento integral por medio de asesorías sobre métodos y hábitos de estudio, preparación de exámenes, consejería académica y talleres para estudiantes que no han cumplido con la normativa de permanencia académica. Estas actividades se realizan de manera grupal y personal.

- Asesoría psicológica a cargo de un equipo de psicólogos que lideran los talleres "Desafío U", atienden las consultas individuales y acompañan a los estudiantes en instancias personales, familiares, sociales y académicas.

- Coaching en sesiones personalizadas que utilizan la metodología coaching de vida para invitar al participante a potencializar sus virtudes y habilidades con el fin de cumplir diferentes metas.

- Ser Pilo Paga tiene como finalidad promover a los estudiantes beneficiarios de Ser Pilo Paga 2 para que continúe en programa estatal y garantice de esta manera la permanencia en la universidad. En este caso, se cuenta con un coordinador que desarrolla diversas acciones, como asignar un padrino, programar encuentros para tratar temáticas específicas que brindan herramientas académicas-personales y hacer seguimiento de notas en cada corte. Específicamente, los padrinos son docentes y/o funcionarios de la universidad que se reúnen esporádicamente durante el semestre con el pilo asignado para escuchar y observar su trayectoria universitaria.

- Tutorías académicas, definidas como el espacio de interlocución entre un docente y un estudiante para aclarar dudas, profundizar sobre un tema y/o reforzar conocimientos. La coordinación de este espacio se encarga de gestionar y programar las reuniones tutoriales en cada escuela, hacer seguimiento del cumplimiento de las tutorías y programar encuentros de tutores. El tutor es un docente de tiempo completo o medio tiempo que tiene dentro de su plan de trabajo la atención tutorial de estudiantes. 


\section{Universidad de ANTIOQUia: ACOMPAÑAmiento CON EQUidAd EN PRO DE} LA FORMACIÓN INTEGRAL

El programa de Permanencia con Equidad surgió con el Plan de Acción 20122015 de la Vicerrectoría de Docencia y por medio de una resolución rectoral, en los cuales se define que es necesario implementar estrategias en relación con el acceso, la permanencia y la graduación de la población estudiantil, así como definir acciones y políticas de inclusión.

Aunque los índices de deserción de la Universidad de Antioquia estaban por debajo del promedio nacional en el año 2012, la institución decidió generar este programa desde la curiosidad en la intervención con las comunidades estudiantiles y además porque encontró que las unidades académicas llevaban a cabo acciones interesantes, pero que estaban desarticuladas respecto al funcionamiento global del centro educativo. En consecuencia, definió como objetivo del programa fortalecer las capacidades institucionales articulando las acciones existentes en cuanto a acceso, permanencia y graduación.

A diferencia de los otros dos programas de acompańamiento mencionados, Permanencia con Equidad no es un ente ejecutor u ordenador de intervenciones tutoriales, sino que se trata de un programa que observa, acompaña, investiga y desea articular las acciones de acompañamiento que ocurren en las 24 unidades de la IES. Tomando en cuenta los ocho componentes del modelo del Ministerio de Educación Nacional para garantizar la permanencia en la educación superior, la Universidad de Antioquia ha trabajado en tres ejes: Observatorio académico, fomento a la permanencia y formación.

Respecto al fomento a la permanencia y la formación, el claustro trabaja en varias líneas: Tutorías, inclusión (diversidad) y banco de iniciativas, que se describen en la figura 3.

Por ser de especial interés para este trabajo, vale la pena profundizar las funciones que desarrolla la institución en la línea de Inclusión:

- Acompañar los procesos de admisión e ingreso en población con discapacidad auditiva o ciega, para lo cual se ajustó la prueba de ingreso implementando el lenguaje braille, audios, textos con letra grande o lenguaje de señas colombiano. Asimismo, los aspirantes en condición de discapacidad auditiva o visual reciben un acompañamiento para que se familiaricen con el examen de admisión.

- Asesorar a las unidades y docentes en los ajustes curriculares y didácticos que deben desarrollar para acompañar la discapacidad. 


\section{TUTORIAS}

- Formalizar en las unidades de pregrado los lineamientos de acción tutorial en relación con su dimensión, metodología, perfil, alcance, funciones y límites de un tutor.

\section{INCLUSIÓN}

- Orientar estrategias que apunten a acompañar a la comunidad estudiantil que puedan estar en mayor riesgo por estar inmersos en una condición o situación vinculada a la marginación.

\section{BANCO DE INICIATIVAS}

- Identificar y acompañar financiera y metodológicamente las propuestas que surgen de los profesores de las diferentes unidades de la Universidad de Antioquia. Asi mismo, escuchar las experiencias de otras universidades y, cuando es viable, desarrollar convenios.

Figura 3. Líneas tutoras del programa de Permanencia con Equidad de la Universidad de Antioquia Fuente: Elaborada por los autores.

- Ofrecer apoyos para facilitar eventos de tipo cultural del cabildo indígena universitario.

- Realizar talleres de sensibilidad a los otros integrantes de la comunidad universitaria, estudiantes, docentes y otros funcionarios, en relación con la inclusión y diversidad.

Ahora bien, en cuanto al componente de Formación, el programa de Permanencia con Equidad ofrece a los tutores, mentores y docentes tres diplomados: Dos que son de nuevo ingreso y uno que es el denominado "Acompañamiento Estudiantil para la Permanencia". En este último se capacita a los participantes sobre qué es un acompañamiento tutorial, cómo se acompaña, las modalidades que existen y la norma vigente en la Universidad de Antioquía. Asimismo, tiene como objetivo que los profesores tengan un norte común, de tal manera que en cada unidad exista un profesional que conozca los lineamientos que se quieren dar y tal vez generen otras acciones que enriquezcan el acompañamiento.

$\mathrm{Al}$ respecto, la directora del programa aclaró en la entrevista que los docentes se forman no necesariamente para actuar como psicólogos o como el papá de un joven, sino que es suficiente con que el profesor tenga conocimiento de dónde remitir a un estudiante de acuerdo con sus necesidades, pues de esta manera se brinda una mirada humana e integral al desarrollo de las tutorías. Finalmente, la 
directora concluye: "Los profesores somos seres humanos que les mostramos a los estudiantes que tenemos un saber y que podemos acompañarlos en el ser".

En el caso de la Universidad de Antioquia se destacan las experiencias de la Escuela de Nutrición, pionera en el tema de tutorías en el país con más de quince años. El programa asigna un tutor por cada semestre y procura que, preferiblemente, tenga a cargo una asignatura. Este docente es un observador y escucha los comportamientos de los estudiantes, interviene cuando existen situaciones que lo ameritan y hace seguimiento a estudiantes que estén en riesgo de pérdida académica. Como parte de sus funciones debe ejecutar la llamada "evaluación cualitativa”, que consiste en una conversación grupal donde se evalúa lo que ha ocurrido desde la matrícula hasta el transcurso del semestre con cada asignatura. Con esta información, todos los tutores del programa se reúnen con la jefe de formación académica de la escuela y reportan los hallazgos más significativos; es decir, constituye una forma de escuchar la voz de los estudiantes y tomar las acciones necesarias. Cabe agregar que en la Escuela también se cuenta con monitores pares que acompañan a estudiantes de primer semestre.

Finalmente, es importante destacar que la experiencia de la universidad se vuelve aún más interesante si se tiene en cuenta que la institución ha realizado investigaciones que caracterizan los procesos de acompañamiento que se desarrollan en las diferentes unidades, fruto de lo cual ha publicado dos libros.

\section{Triangulación de los resultados}

La triangulación de datos es fundamental para esta fase de la investigación, pues los datos obtenidos en las entrevistas a los directores del programa y a los docentes de cada IES arrojaron información valiosa sobre las acciones teóricas y metodológicas, así como sobre la opinión de los entrevistados en cuanto a perfil del tutor y los beneficios que obtienen los estudiantes que participan en el acompañamiento. Estos resultados se analizaron inicialmente de forma individual y luego se hizo un contraste entre las tres IES para determinar sus similitudes o diferencias y, de esta manera, fundamentar una teoría que describa el actual estado de los acompañamientos y sus características estructurales y funcionales. Con estos en insumos, la siguiente fase de la investigación puede proponer un modelo que brinde una mirada integral a los jóvenes inmersos en la educación superior.

Ahora bien, para comprender la triangulación de datos versus la propuesta de un modelo de acompañamiento es necesario analizar la información que se presenta en la figura 4: 


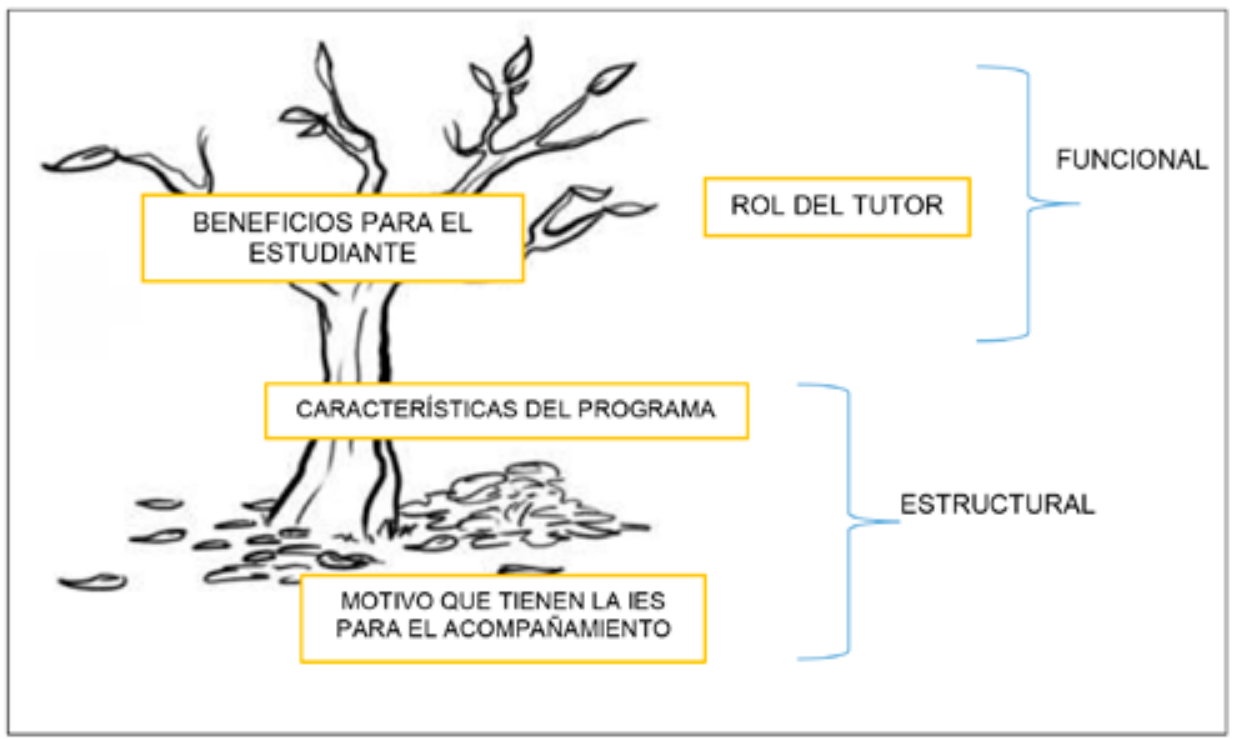

Figura 4. Modelo propuesto para el acompañamiento integral de estudiantes universitarios - triangulación de datos entrevista actores del acompañamiento Fuente: Elaborada por los autores.

La figura 4 metaforiza el árbol de la vida y sugiere que el sostén de los acompañamientos a estudiantes universitarios (raíces del árbol) es el motivo o la intención de las instituciones para realizar estas acciones, que se dimensiona en las características propias del programa (tronco) y que deriva en unas hojas y frutos que configuran el perfil de un tutor y benefician a los estudiantes participantes.

A continuación, se presentan las rejillas que se diseñaron para hacer la triangulación en relación con las cuatro categorías enunciadas: (1) Características del programa, (2) intención de las IES con la realización de las acciones tutoriales, (3) perfil del profesional que acompaña y (4) beneficios para el estudiante participante. 


\section{CARACTERÍSTICAS DEL PROGRAMA}

Dado que estas se mencionaron en el apartado anterior, la rejilla solo contiene la información básica (tabla 1):

Tabla 1. Características del programa

\begin{tabular}{|c|c|c|c|}
\hline Ítem & $\begin{array}{c}\text { Universidad } \\
\text { Sergio Arboleda }\end{array}$ & $\begin{array}{l}\text { Universidad de } \\
\text { Antioquia }\end{array}$ & Universidad del Rosario \\
\hline $\begin{array}{l}\text { Nombre del } \\
\text { programa }\end{array}$ & No refiere & $\begin{array}{l}\text { Permanencia con } \\
\text { Equidad }\end{array}$ & $\begin{array}{l}\text { Programa de } \\
\text { Acompańamiento para } \\
\text { Todos (Pacto) }\end{array}$ \\
\hline $\begin{array}{l}\text { Dependencia } \\
\text { que le dirige }\end{array}$ & $\begin{array}{l}\text { Decanatura } \\
\text { de estudiantes }\end{array}$ & $\begin{array}{l}\text { Vicerrectoría } \\
\text { de Docencia }\end{array}$ & $\begin{array}{l}\text { Dirección } \\
\text { de Estudiantes }\end{array}$ \\
\hline Servicios & $\begin{array}{l}\text { Portafolio de servicios } \\
\text { ofertado a los estudiantes } \\
\text { de las escuelas. }\end{array}$ & $\begin{array}{l}\text { Basada en componentes } \\
\text { del MEN para la perma- } \\
\text { nencia en educación } \\
\text { superior: } \\
\text { - Fomento a la perma- } \\
\text { nencia, en las líneas } \\
\text { de Tutorías, Inclusión } \\
\text { (diversidad) y Banco de } \\
\text { Iniciativas. } \\
\text { - Formación a docentes } \\
\text { mediante tres diplo- } \\
\text { mados. }\end{array}$ & $\begin{array}{l}\text { - Nombramiento de un } \\
\text { director de cohorte en } \\
\text { cada unidad que puede } \\
\text { contar con un tutor en } \\
\text { cada programa. } \\
\text { - Seminario de contex- } \\
\text { tualización. } \\
\text { - Cuestionario de } \\
\text { Caracterización. } \\
\text { - Reunión de docentes. }\end{array}$ \\
\hline
\end{tabular}

Fuente: Elaborada por las autoras.

\section{SiMILITUdES ENTRE LOS PROGRAMAS}

A continuación, se sintetizan las principales similitudes entre los programas, de acuerdo con la triangulación de la información suministrada en las entrevistas:

- En las tres IES existen acciones que se ofertan a los estudiantes para atender las necesidades que tengan en su trayectoria de vida universitaria. Asimismo, hacen un acompañamiento desde el primer semestre, incluyendo la aplicación de un instrumento de caracterización. Particularmente, la Universidad de Antioquía amplía las acciones tutoriales en el proceso de admisión de aspirantes con discapacidad.

- Los actores del acompañamiento varían de acuerdo con la estructura del programa que ha definido cada institución. Se identifican figuras como: Mentor, tutor, telemano, padrino, director de cohorte, asesor. En las tres universidades se promueve el acompañamiento entre pares, 
descrito como un grupo de estudiantes orientados a la asesoría académica o consejería de la vida universitaria.

- En las tres IES se posee una estructura jerárquica cuya cabeza es el director general, que está a cargo de un grupo de coordinadores por área o cohorte. En el caso de la Universidad de Antioquía, el administrador del programa es la Vicerrectoría Docente, con un director que gestiona para toda el alma máter y un comité en cada unidad, que está conformado por vicedecano, profesional de bienestar, representante profesoral y un representante de estudiantes y otro profesional.

\section{Contraposiciones de los PROgramas}

En esta sección se da cuenta de los aspectos en los que divergen las IES investigadas:

- Definición: No existe una nominación y definición estándar para las acciones de acompańamiento de cada IES. En el caso de la Universidad de Antioquia, cualquier tipo de acompańamiento que se realice al estudiante es una acción tutorial, mientras que en el Rosario y en la Sergio Arboleda se plantean figuras como mentor, tutor y director de cohorte.

- Estructura: Las universidades del Rosario y Sergio Arboleda poseen programas que intervienen directamente en la población de pregrado, mientras que en la de Antioquia, el programa Permanencia con Equidad surge como un plan de acción rectoral que tiene como objetivo replantear las acciones existentes de las 24 unidades académicas y administrativas de la institución y así articular las acciones en pro del acceso, permanencia y graduación.

- Proceso: Las acciones tutoriales de las universidades del Rosario y Antioquia en la Facultad de Comunicación y en la Escuela de Nutrición, respectivamente, han estructurado una metodología en la cual los estudiantes reciben un acompańamiento por cohorte y/o por semestre, de manera que se trata de un proceso transversal y continúo que permite hacer seguimiento a todas las instancias de la vida universitaria. Además, estas acciones generan una red de apoyo en cada unidad que incluye pares, docentes, funcionarios administrativos y de servicios generales, por lo cual el acompañamiento no es responsabilidad de un director de corte o del tutor asignado, sino que es parte de una cultura institucional. 


\section{Intención o motivo de realización de programas de acompańamiento}

Este apartado sintetiza los análisis de la información que arrojó la siguiente pregunta: ¿Por qué considera que la IES realiza este tipo de acompañamientos? La tabla 2 muestra los resultados por entrevistado y por institución.

Tabla 2. Intención o motivo de las IES al realizar el acompańamiento

\begin{tabular}{|c|c|c|c|}
\hline Entrevistado & $\begin{array}{c}\text { Universidad Sergio } \\
\text { Arboleda }\end{array}$ & $\begin{array}{l}\text { Universidad de } \\
\text { Antioquia }\end{array}$ & $\begin{array}{l}\text { Universidad del } \\
\text { Rosario }\end{array}$ \\
\hline $\begin{array}{l}\text { Profesional } \\
\text { que realiza el } \\
\text { acompańa- } \\
\text { miento }\end{array}$ & $\begin{array}{l}\text { - Promover la retención } \\
\text { de estudiantes y evitar } \\
\text { la deserción. } \\
\text { - Facilitar el desarrollo } \\
\text { de un rol docente } \\
\text { visto como un adulto } \\
\text { cercano que se rela- } \\
\text { ciona con el estudiante } \\
\text { en la confianza y el } \\
\text { compañerismo. }\end{array}$ & $\begin{array}{l}\text { - Posibilitar la perma- } \\
\text { nencia con bienestar, } \\
\text { calidad y acompaña- } \\
\text { miento integral. }\end{array}$ & $\begin{array}{l}\text { - Cumplir con los prin- } \\
\text { cipios rosaristas de } \\
\text { humanismo integral. } \\
\text { - Posibilitar la perma- } \\
\text { nencia. }\end{array}$ \\
\hline $\begin{array}{l}\text { Director del } \\
\text { programa }\end{array}$ & $\begin{array}{l}\text { - Cumplir con los prin- } \\
\text { cipios humanistas de la } \\
\text { universidad. } \\
\text { - Posibilitar la perma- } \\
\text { nencia. }\end{array}$ & $\begin{array}{l}\text { - Responsabilidad social } \\
\text { que acompaña la } \\
\text { misión de la univer- } \\
\text { sidad. } \\
\text { - Permitir la graduación } \\
\text { oportuna. }\end{array}$ & $\begin{array}{l}\text { - Acoger y conocer a sus } \\
\text { estudiantes. } \\
\text { - Acompañamiento inte- } \\
\text { gral por misionalidad } \\
\text { de la IES. } \\
\text { - Lograr permanencia y } \\
\text { graduación, pero más } \\
\text { que una estadística, se } \\
\text { busca que el estudiante } \\
\text { cumpla el objetivo } \\
\text { que se ha planteado al } \\
\text { ingresar a la IES. }\end{array}$ \\
\hline
\end{tabular}

Fuente: Elaborada por los autores.

Al indagar a los entrevistados sobre la intención o el motivo por el cual la institución realiza este tipo de acompañamientos, se encontró que en las tres IES la misión de la universidad se relaciona con el objetivo del programa. De manera particular, la Universidad Sergio Arboleda respondió que su motivación para desarrollar el programa es cumplir con los principios humanistas-cristianos, posibilitar la permanencia y evitar la deserción, posibilitar un rol docente que le permita ser un adulto cercano que se relaciona con el estudiante en la confianza y compańerismo. Por su parte, la Universidad del Rosario afirmó que su interés es cumplir con los principios rosaristas de humanismo integral, el cual busca acoger y conocer a los 
estudiantes, posibilitar la permanencia y graduación, pero también advierte que más que una estadística, se espera que el estudiante cumpla el objetivo que se ha planteado al ingresar a la IES. Finalmente, la Universidad de Antioquia señaló los siguientes motivos para formular el acompañamiento: La responsabilidad social que acompaña la misión de la universidad y que busca la permanencia con bienestar y calidad, junto con una graduación oportuna y por medio de un acompańamiento integral.

Para concluir, es importante destacar cómo las universidades del Rosario y de Antioquia coinciden en las respuestas de los entrevistados y entre ellas en términos del acompañamiento integral, la permanencia y la graduación con calidad y proyecto de vida del estudiante.

\section{Beneficios para el estudiante que participan en el acompañamiento}

A continuación, la tabla 3 sintetiza las respuestas que dieron los entrevistados sobre los beneficios que obtienen los estudiantes que participan en los programas descritos.

Tabla 3. Beneficios que obtiene el estudiante

\begin{tabular}{|c|c|c|c|}
\hline Actor & $\begin{array}{c}\text { Universidad Sergio } \\
\text { Arboleda }\end{array}$ & $\begin{array}{c}\text { Universidad de } \\
\text { Antioquia }\end{array}$ & $\begin{array}{c}\text { Universidad del } \\
\text { Rosario }\end{array}$ \\
\hline $\begin{array}{l}\text { Profesional que } \\
\text { realiza el acompaña- } \\
\text { miento }\end{array}$ & $\begin{array}{l}\text { - Apropiación del } \\
\text { conocimiento, visto } \\
\text { como la aplicación } \\
\text { y transferencia de } \\
\text { lo que está apren- } \\
\text { diendo. } \\
\text { - Despertar la crea- } \\
\text { tividad, pues se } \\
\text { entiende cómo } \\
\text { funcionan las herra- } \\
\text { mientas dadas. }\end{array}$ & $\begin{array}{l}\text { - Superar las situa- } \\
\text { ciones de riesgo que } \\
\text { atraviesan. } \\
\text { - Ser un egresado } \\
\text { integral con carac- } \\
\text { terísticas personales } \\
\text { sobresalientes. }\end{array}$ & $\begin{array}{l}\text { - La formación } \\
\text { integral, pues les } \\
\text { permite verse en su } \\
\text { humanidad y cómo } \\
\text { pueden ser compa- } \\
\text { sivos y solidarios con } \\
\text { el otro. }\end{array}$ \\
\hline $\begin{array}{l}\text { Director del } \\
\text { programa }\end{array}$ & $\begin{array}{l}\text { - Reconocimiento de } \\
\text { la universidad como } \\
\text { una institución } \\
\text { humanista. } \\
\text { - La calidad humana, } \\
\text { amabilidad y cordia- } \\
\text { lidad que encuentra } \\
\text { en la decanatura. }\end{array}$ & $\begin{array}{l}\text { - Potenciar en el estu- } \\
\text { diante el desarrollo } \\
\text { humano. } \\
\text { - Formación como } \\
\text { ciudadanos y profe- } \\
\text { sionales integrales. }\end{array}$ & $\begin{array}{l}\text { - Lograr afrontar satis- } \\
\text { factoriamente las } \\
\text { diferentes etapas de } \\
\text { la vida universitaria. }\end{array}$ \\
\hline
\end{tabular}

Fuente: Elaborado por los autores. 
Como se observa en la tabla 3, este ítem arrojó dos tipos de respuestas. Por una parte, la Universidad Sergio Arboleda, en la voz de un tutor académico, sostuvo que el mayor beneficio es la apropiación del conocimiento, entendido como la aplicación y transferencia de lo que está aprendiendo. Mientras que la decana de Estudiantes consideró que el acompañamiento es una oportunidad para reconocer que la universidad es humanista y posee calidad humana. Por otra parte, las universidades del Rosario y de Antioquia coinciden que el estudiante participante del acompańamiento logra afrontar y superar las etapas de la vida universitaria, incluyendo los riesgos que esta puede presentar. En el caso de la Universidad de Antioquia, la institución enfatiza en potenciar el desarrollo humano y la formación de profesionales integrales, mientras que la del Rosario considera que el acompañamiento permite a los estudiantes observarse en su humanidad y cómo pueden ser compasivos y solidarios con otros. En otras palabras, la Universidad de Antioquia y la del Rosario se refieren de manera continua en términos de acompañamiento integral.

\section{Perfil del profesional que realiza el acompañamiento}

Siguiendo con la exposición, a continuación, se presentan los hallazgos sobre el perfil profesional que se espera de las personas que hacen el acompañamiento (tabla 4).

Tabla 4. Perfil del profesional que realiza el acompañamiento

\begin{tabular}{|c|c|c|c|}
\hline Actor & $\begin{array}{c}\text { Universidad Sergio } \\
\text { Arboleda }\end{array}$ & $\begin{array}{l}\text { Universidad de } \\
\text { Antioquia }\end{array}$ & $\begin{array}{l}\text { Universidad del } \\
\text { Rosario }\end{array}$ \\
\hline $\begin{array}{l}\text { Profesional que realiza } \\
\text { el acompañamiento }\end{array}$ & $\begin{array}{l}\text { - Disposición en } \\
\text { romper la barrera } \\
\text { que en ocasiones se } \\
\text { establece entre el } \\
\text { profesor y el estu- } \\
\text { diante. } \\
\text { - Mente abierta, } \\
\text { mirada comprensiva, } \\
\text { disposición, aceptar } \\
\text { al otro. }\end{array}$ & $\begin{array}{l}\text { - Empatía, cercanía } \\
\text { a los estudiantes, } \\
\text { sensibilidad ante las } \\
\text { situaciones del otro, } \\
\text { entender que hay un } \\
\text { ser humano detrás } \\
\text { del estudiante. } \\
\text { - Flexible, compren- } \\
\text { sivo, entender las } \\
\text { particularidades del } \\
\text { grupo y de cada } \\
\text { estudiante. } \\
\text { - En el aspecto profe- } \\
\text { sional: Experiencia } \\
\text { en el quehacer peda- } \\
\text { gógico. }\end{array}$ & $\begin{array}{l}\text { - Disposición para el } \\
\text { acompañamiento. } \\
\text { Comprensión del } \\
\text { proceso adoles- } \\
\text { cente y entender el } \\
\text { impacto que tiene el } \\
\text { acompañamiento en } \\
\text { la vida de un estu- } \\
\text { diante. }\end{array}$ \\
\hline
\end{tabular}




\begin{tabular}{|c|c|c|c|}
\hline Actor & $\begin{array}{c}\text { Universidad Sergio } \\
\text { Arboleda }\end{array}$ & $\begin{array}{l}\text { Universidad de } \\
\text { Antioquia }\end{array}$ & $\begin{array}{l}\text { Universidad del } \\
\text { Rosario }\end{array}$ \\
\hline Director & $\begin{array}{l}\text { - Profesional que } \\
\text { conozca el tema } \\
\text { curricular de los } \\
\text { estudiantes, que } \\
\text { haya trabajado en } \\
\text { gestión social estu- } \\
\text { diantil. } \\
\text { - Alguien que sepa } \\
\text { llegarle al estudiante. } \\
\text { - Ser una persona } \\
\text { humanista y } \\
\text { comprender el } \\
\text { concepto de la } \\
\text { universidad. }\end{array}$ & $\begin{array}{l}\text { - Alguien que vaya } \\
\text { más allá de lo acadé- } \\
\text { mico y que pueda } \\
\text { reconocer al estu- } \\
\text { diante más allá de lo } \\
\text { académico. }\end{array}$ & $\begin{array}{l}\text { - Alguien que vivan } \\
\text { el acompañamiento, } \\
\text { se perciba cercano } \\
\text { a los estudiantes y } \\
\text { a los intereses del } \\
\text { programa. }\end{array}$ \\
\hline
\end{tabular}

Fuente: Elaborada por los autores.

Estos resultados son interesantes en cuanto a que las manifestaciones de los seis entrevistados coinciden en los siguientes aspectos:

- Disposición para el acompañamiento y para estar con el otro (en este caso el estudiante) desde la empatía, aceptación, comprensión y sensibilidad.

- $\quad$ Romper la barrera que pueda existir entre el docente y el estudiante yendo más allá de lo académico, entendiendo las particularidades del grupo y de cada estudiante para generar una relación flexible y cercana.

- Comprensión del concepto de la universidad, del acompañamiento y de su impacto.

\section{Conclusiones}

En correspondencia con los objetivos planteados, se confirma que la tutoría es un espacio de atención al estudiante donde se le acompaña en su proceso de formación y aprendizaje, de manera que logra establecer un vínculo de confianza y apoyo con el docente.

Igualmente, la mayoría de los acompañamientos tutoriales demuestran que gracias a este ejercicio los estudiantes se empoderan de sus habilidades y estrategias de aprendizaje, con lo cual, además, mejoran o mantienen sus rendimientos 
académicos. Por esta razón, las IES llevan a cabo acciones tutoriales encaminadas a acompañar a los estudiantes y asegurar así su permanencia en la educación superior.

Se concluye también que dependiendo de cómo se encaminen institucionalmente los programas, las tutorías se pueden definir por categorías y modalidades. Además, se estableció que los planes de acciones tutoriales ofrecen una estructura organizada y enfocada a cumplir los objetivos que se proponen las universidades con los acompañamientos a estudiantes. En este sentido, la planeación de estos programas se debe basar en estudios previos de caracterización de los estudiantes, así como contar con el apoyo de todos los participantes de la institución educativa y los entes gubernamentales. El trabajo en equipo, la comunicación asertiva y la evaluación transversal y continúa son aspectos que pueden garantizar su éxito.

En cuanto a los beneficios que reciben los estudiantes que acceden a los acompañamientos, cabe afirmar que los profesionales que ejecutan y dirigen el acompańamiento seńalan que estos logran afrontar y superar las etapas de la vida universitaria, a la vez que reciben formación integral que se deriva en su desarrollo personal y profesional. En este sentido, las acciones tutoriales se convierten en la excusa perfecta para romper las barreras que pueden existir en el aula, ya que la tutoría posibilita que la mirada que tiene el docente sobre el estudiante traspase un código o una calificación, que lo valore desde sus logros y retos, al tiempo que crea las condiciones para que se muestre cercano y empático para que el educando interactúe en sus entornos desde la colaboración.

En consecuencia, un programa de acompańamiento a estudiantes universitarios de tipo estructural debe partir de un interés de las IES en la formación integral, el humanismo, la responsabilidad social y la permanencia estudiantil, en otras palabras, en una preocupación por ejercer la educación en términos de equidad, participación e inclusión.

Otra conclusión es que las universidades analizadas asignan como parte del rol de tutor la tarea de desarrollar acciones de tipo académico o personal y que convierten al docente o par en una figura cercana que acompaña al estudiante en su vida universitaria. Adicionalmente, estas instituciones dirigen sus acciones tutoriales a los estudiantes de pregrado con la meta de acompañar las diferentes instancias de la vida universitaria. Especial interés despierta la población de primer semestre, que se caracteriza a través de distintos instrumentos para identificar sus necesidades.

Finalmente, en relación con los modelos que se identifican en las tres universidades, se evidenció que la misión de la institución constituye la base de los programas de acompañamiento. $\mathrm{Y}$ aunque es necesario advertir que deben forta- 
lecer su estructuración, específicamente la funcionalidad, se reconoce como positivo que hay experiencias significativas y particularidades en cada centro educativo, como las diferencias de los acompañamientos según los programas y las disciplinas de formación de los tutores. Para concluir, los análisis evidencian que es fundamental que las IES articulen las intenciones entre los tutores y las unidades académicas que desarrollan las tutorías.

\section{Bibliografía}

\section{Obras citadas}

Bagnis, G. (2003). Programa institucional de tutorías [documento en línea]. Recuperado de http:// campusdigital.uag.mx/academia/modelo/PI_Tutorias.pdf

Castillo A., Torres, G. \& Polanco, G. (2009). Tutoría en la enseñanza y la empresa. Madrid: Pearson Educación.

Flick, U. (2014). La gestión de la calidad en investigación cualitativa. Madrid: Morata.

Gómez, C. (2012). Percepción de los estudiantes sobre el programa de tutoría académica. Convergencia, 58, 209-233. Recuperado de http://www.scielo.org.mx/pdf/conver/v19n58/v19n58a9.pdf

Hernández, S., Fernández, C. \& Baptista, P. (2006). Metodología de la investigación. México: McGraw Hill.

Ladino (2004). La tutoría Académica. Cuadernos de Psicopedagogía. En http://www.uptc.edu.co/ facultades/f_educacion/pregrado/psicopedagogia/do.

Molina, I. (2012). Estado del arte sobre tutorías. Revista Civilizar, 12 (22), 167-176. Recuperado de http://www.scielo.org.co/pdf/ccso/v12n22/v12n22a12.pdf

Molina, I. \& Gallardo, I. (2011). Las tutorías académicas: Una necesidad de impulsar los estilos participativos y demostrar cómo influyen en la formación de profesores. En Varios Autores, La pedagogia colombiana. Educación, pedagogía y curriculo (t. 1) (pp. 293-300). Bogotá, D. C.: Redipe.

Monge, Crespo, C, (2009). Tutoría y Orientación Educativa; Nuevas Competencias. En https:// www.academia.edu/4318683/MONGE_CRESPO_C._2009_._Tutor\%C3\%ADa_y_ Orientaci\%C3\%B3n_Educativa_Nuevas_competencias

Peinado-Guevara, H. G., Peinado-Guevara, V. M., Huerta-Sandoval, J. A., Mendoza-Zamora, F. \& Ladron, M. de los A. (2011). Impacto de la tutoría escolar en la proyección de los estudiantes adultos en el nivel medio superior, modalidad semiescolar. Ra Ximhai, 7 (1), 21-31. Recuperado de http://www.revistas.unam.mx/index.php/rxm/article/view/26663/24980

Rodríguez, F. \& Sierra, U. (2014). Reflexiones pedagógicas sobre la tutoría académica en educación superior. Revista Salud Bosque, 4 (1), 29-36.

Rodríguez, Gil. \& García J. (2007). Metodología de la investigación Cualitativa. Ediciones Aljibe.

Sánchez, M. (2006). Cuadernos de psicopedagogía No. 3. La tutoría académica. Universidad Pedagógica y Tecnológica de Tunja. Ciencias de la Educación, en https://revistas.uptc.edu.co/ index.php/psicopedagogia/article/view/497. 
Trejo, M. (2010). Fenomenología como método de investigación: Una opción para el profesional de enfermería. Revista. Enf neurol, 11 (2), 98-101. Recuperado de http://www.medigraphic.com/ pdfs/enfneu/ene-2012/ene122h.pdf

Valles Ruiz, R. M., Torres Cuevas, M. E. \& Godínez Guzmán, H. M. (2012). La acción tutoría en la Universidad Autónoma de Hidalgo en el Instituto de Ciencias Sociales y Humanidades. Ponencia presentada en el V Encuentro Nacional de Tutorías. Universidad Autónoma del Estado de Hidalgo, México. Recuperado de https://www.uaeh.edu.mx/investigacion/productos/4965/ ponencia_tutoria_sonora_29_ago_12_-_2.pdf

\section{Obras consultadas}

Aigneren, M. (2009). La técnica de recolección de información mediante los grupos focales. La Sociología en sus Escenarios [revista electrónica], 7 (20). Recuperado de http://aprendeenlinea. udea.edu.co/revistas/index.php/ceo/article/viewFile/1611/1264

Arcade, J., Godet, S., Meunier, F. \& Roubelat, C. (2004). Análisis estructural con el método Micmac y estrategia de los actores con el método Mactor [documento en línea]. Recuperado de https:// docplayer.es/1594804-Analisis-estructural-con-el-metodo-micmac-y-estrategia-de-los-actorescon-el-metodo-mactor.html

Bueno, G. (2005). Perfil sociodemográfico de los estudiantes universitarios sin progreso académico y la relación entre el apoyo psicosocial con enfoque de resiliencia y el éxito académico en estudiantes de primer año. (Tesis de doctorado). Universidad Complutense Madrid, España.

Davis, S. \& Palladino, J. (2008). Psicología. Pearson Prentice Hall. México.

Flick, U. (2004). Introducción a la investigación cualitativa. Madrid: Morata.

García, I., Cuevas, S., Vales, G. \& Cruz, M. (2012). Impacto del Programa de Tutoría en el desempeño académico de los alumnos del Instituto Tecnológico de Sonora. Revista Electrónica de Investigación Educativa, 14, 106-121.

Garrote, R. (2015). La validación por juicio de expertos: Dos investigaciones cualitativas en lingüística aplicada. Revista Nebrija de Lingüistica Aplicada a la Enseñanza de Lenguas, 18. Recuperado de https://www.nebrija.com/revista-linguistica/files/articulosPDF/articulo_55002aca89c37.pdf

Giandoni, O. \& Moraes, H. (2012). Concepçóes de validade em pesquisas qualitativas. Educação e Pesquisa, 38 (1), 229-241. Recuperado de http://www.scielo.br/pdf/ep/v38n1/ep448.pdf

Prieto, M. \& March, C. (2002). Paso a paso en el diseño de un estudio mediante grupos focales. Aten Primaria, 29 (6), 366-373. Recuperado de https://ac.els-cdn.com/S0212656702705854/1s2.0-S0212656702705854-main.pdf?_tid=ce60b9e3-7a53-440a-934e-b262695f4c64\&acdna$\mathrm{t}=1543531355$ 59db72af84c4715f747bb48ed532c195

Quintana, A. y Montgomery, W. (Eds.) (2006). Metodología de la Investigación Científica Cualitativa. Psicología: Tópicos de actualidad .Lima: UNMSM.

. Sánchez, F. \& Márquez, J. (2012). La deserción en la educación superior en Colombia durante la primera década del siglo XXI: ¿Por qué ha aumentado tanto? [Documentos CEDE]. Bogotá, D. C.: Uniandes. 


\section{RECAPITULACIÓN}

Este apartado de cierre no es una síntesis, tampoco un epílogo en todo el sentido de la palabra, pues no constituye un discurso conclusivo de una obra terminada, ya que tampoco el escrito lo es. Es una introversión dirigida a quienes, como los que escriben, son educadores preocupados e interesados por producir nuevas ideas y cambios en la educación científica a través de la investigación formativa en las universidades, así como por recuperar la acción de crear soluciones desde las ciencias de la educación y la pedagogía a las problemáticas actuales. En términos pedagógicos, es una "recapitulación de la idea central que los autores quieren retomar".

Los resultados expuestos no son concluyentes, como sucede en la ciencia. Solo el continuum del conocimiento coherente, desde las tradiciones y la búsqueda de la objetividad con la mayor certeza, permite alcanzar la mejor propuesta de solución, que solo se logra a través de aproximaciones sucesivas de la comunidad científica, para el caso en la academia. Estamos convencidos de que falta mucho por pensar y hacer para formar profesionales con pensamiento crítico, lógico e interesado en resolver los problemas desde el conocimiento científico.

Para los autores, es apremiante investigar los procesos educativos con el objetivo de que las nuevas propuestas se fundamenten en el rigor científico; solo así los debates sobre la investigación formativa y las tutorías de trabajo de grado (tema central del libro) tendrán la altura necesaria para contribuir al desarrollo de la formación integral. En este sentido, es imperioso asumir la ciencia de la educación y la pedagogía en la formación de profesionales para poder argumentar el lugar y la especificidad de la profesión del educador, la cual debe ser conocida por quienes se integran a tan elevada labor cultural. Aunque hoy nos referimos al nuevo rol del profesor como tutor, el desconocimiento sobre su significado impide lograr esta transformación; no se trata de trasladar buenas intenciones, sino de que nuestra cultura educativa avance decididamente

En consecuencia, este libro no solo pretende que los lectores cuestionen su accionar pedagógico ante la investigación formativa, sino que además hagan un examen introspectivo y valorativo que incorpore a su quehacer la investigación educativa de los procesos de educación científica, la cual es transversal a todas las disciplinas y ciencias que enseñamos. 


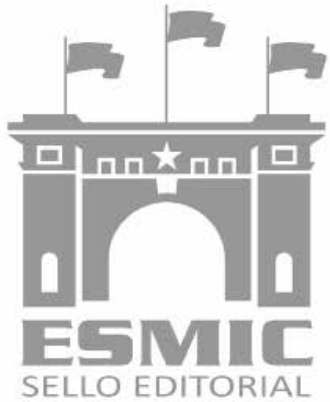

